\title{
El uso de la gamificación para el fomento de la educación inclusiva
}

Recibido: 14 de abril de 2019 / Revisado: 20 de abril de 2019

Aceptado: 31 de mayo de 2019 / Publicado: 22 de julio de 2019

CARMEN RODRÍGUEZ JIMÉNEZ

Departamento Didáctica y Organización Escolar, Universidad de Granada, España rodri96@correo.ugr.es

MAGDALENA RAMOS NAVAS-PAREJO

Departamento Didáctica y Organización Escolar, Universidad de Granada, España magdalenarnp@correo.ugr.es

MARÍA JESÚS SANTOS VILLALBA

Departamento Didáctica y Organización Escolar, Universidad Internacional de la Rioja, España mariajesus.santos@unir.net

JUAN MIGUEL FERNÁNDEZ CAMPOY

Departamento Didáctica y Organización Escolar, Universidad de Granada, España jmcampoy@ugr.es

\section{RESUMEN}

La gamificación y su aplicación a la educación es una tendencia cada vez más implantada en las aulas de todos los niveles educativos. Al mismo tiempo, la inclusión de todo el alumnado es una temática de relevancia en la literatura científica y que sigue siendo objeto de debate e investigación. Con este artículo se quiere poner de relieve el uso de la gamificación como un medio para fomentar la inclusión del alumnado en general, y del alumnado que presenta algún tipo de NEAE (Necesidades Específicas de Apoyo Educativo), en particular. Reconocemos en la inclusión educativa un modelo de actuación que apuesta por ofrecer una enseñanza de calidad a todo el alumnado y que identifica en la diversidad, un valor inherente y enriquecedor. La revisión

\section{ABSTRACT}

The use of gamification for the promotion of the inclusive education

Gamification and its application to education is a growing trend in classrooms at all levels of education. At the same time, the inclusion of all students is an issue of relevance in scientific literature and continues to be the subject of debate and research. The aim of this article is to highlight the use of gamification as a means of promoting the inclusion of students in general, and of students with some type of SEND (Special Educational Needs and Disabilities), in particular. We recognise that educational inclusion is a model of action that is committed to offering quality education to all students, 
bibliográfica realizada pone de manifiesto las ventajas que supone la gamificación como herramienta facilitadora de la inclusión educativa y la necesidad de ir introduciendo esta metodología activa en las aulas, todo ello, con el fin de conseguir una mayor motivación e implicación por parte de los docentes y discentes en el proceso de enseñanza-aprendizaje.

Palabras Clave: Aprendizaje basado en juegos, Educación Inclusiva, Métodos de Enseñanza, Aprendizaje Activo; and that identifies diversity as an inherent and enriching value. The bibliographical review carried out highlights the advantages of gamification as a tool to facilitate educational inclusion and the need to introduce this active methodology into the classroom, all with the aim of achieving greater motivation and involvement on the part of teachers and students in the teaching-learning process.

Keywords: Service Learning, school-community relationship, community participation.

\section{Introducción}

En los últimos años, en el marco de la atención a la diversidad y de la inclusión educativa, el alumnado adquiere un mayor protagonismo en su aprendizaje y el profesorado precisa de dotarse de la capacidad de controlar, analizar e investigar nuevas estrategias metodológicas, así como de reinventar su propia práctica docente, para dar respuesta a todo su alumnado (Fernández-Batanero, 2018).

Tradicionalmente, en los escenarios educativos se recurría a una enseñanza expositiva de corte tradicional, identificándose en el docente un mero transmisor de contenidos, y en el discente un receptor pasivo de los mismos. Las corrientes educativas actuales evidencian la necesidad de fomentar una plena participación y protagonismo del alumnado en la construcción de conocimientos y aprendizajes, convirtiéndose el docente en un mediador entre el estudiante y su proceso formativo (Serrano \& Pons, 2011).

Las reformas en la enseñanza en las que estamos sumergidos y la gran diversidad de alumnado que hay dentro de las aulas, nos exhortan a desarrollar otras metodologías que den una respuesta adecuada y ajustada a las necesidades de cada alumno (Jerez, 2015; León \& Crisol, 2011). Por ello, los docentes deben convertirse en facilitadores de entornos inclusivos para que así, la educación que se oferte sea de calidad para todos y cada uno de los agentes implicados (Ruiz-Palmero \& Sánchez-Rodríguez, 2017).

El cambio de rol que el profesorado debe asumir se basa en mediar en las diferentes situaciones que se presenten en el aula, para lograr los mismos objetivos en un contexto de equidad y calidad educativa. De la misma manera que debe asumir la incorporación de las TIC para la mejora de la educación y la inclusión del alumnado, por el alto nivel igualador de oportunidades que poseen, si se tiene en cuenta que facilitan la comunicación e interacción, independientemente de las limitaciones del alumnado (Fernández-Batanero, 2018). 
La educación inclusiva, se entiende como un proceso que ha de sustentarse en un cambio de paradigma fundamentado en una ideología y unos planteamientos de intervención asentados en valores de derecho, calidad y equidad, que debe estar presente en la mentalidad de docentes, familias y sociedad en su conjunto (Muntaner, 2019)

Para poder desarrollar la competencia social y cívica favoreciendo la inclusión, Rodríguez y Arroyo (2014) establecen que los videojuegos son una herramienta imprescindible a la hora de potenciar los aspectos que tienen que ver con esta competencia. Además, esta herramienta promueve el entretenimiento y la motivación, algo que supone que la función socializadora aumente. Además, los estudiantes en la actualidad dedican gran parte de su tiempo de ocio a los videojuegos o a otras actividades lúdicas. Si partimos de esta relación de los jóvenes de diferentes edades con los videojuegos, se puede suponer que la aplicación de estas herramientas dentro del aula pueda surtir un efecto positivo en la motivación y, por tanto, en el aprendizaje de los alumnos.

Así, llegamos al convencimiento actual que expone la necesidad imperativa de cambiar los procesos de Enseñanza-Aprendizaje (E-A) hasta ahora conocidos. Esto es, por tanto, una renovación metodológica en la que nos encontramos inmersos y que afecta a todos los niveles educativos (Fernández, 2012; Robledo, et al., 2015).

\section{Estado actual de la inclusión educativa}

En 1966, el Informe Coleman titulado "Equality of Educational Opportunity", reflejó la importancia de eliminar las desigualdades existentes dentro del ámbito educativo. Este mismo informe animaba a poner el foco de atención en una mejora de la educación de los grupos minoritarios para que estos se integraran (García, 2015).

Coleman en el año 1968 señaló que, la situación socioeconómica y cultural de las familias son factores determinantes en la educación y que la escuela en lugar de subsanar diferencias de esa índole, las acentúa.

El término "inclusión” fue acuñado por el informe Warnock en el año 1978 (Perazzo \& Gargiulo, 2009). Este concepto nace del modelo de discapacidad y considera que los niños y niñas son diferentes entre sí. Pero no es hasta el año 1990, cuando fue introducido en el sistema educativo español dicho término, el cual está reflejado en la Ley Orgánica General del Sistema Educativo (LOGSE).

Desde el año 2000, la Organización para la Cooperación y el Desarrollo Económico (OCDE) viene realizando estudios sobre las competencias alcanzadas en los estudiantes que se encuentran próximos 
a finalizar la enseñanza obligatoria; los conocidos informes PISA que se realizan trianualmente y ofrecen una importante información sobre la calidad de los sistemas educativos y la formación del capital humano, factor determinante del progreso de las sociedades (Villar, 2018). El alumnado en esta prueba realiza test no solo sobre conocimientos, además completan un exhaustivo cuestionario relativo a su entorno familiar, hábitos de estudio, actitudes y motivación, con el fin de obtener información sobre los resultados en función de las variables económicas, demográficas y sociales para poder diseñar políticas de mejora encaminadas a favorecer la inclusión. Existen tres variables que miden distintos aspectos de los resultados obtenidos:

- El rendimiento, que se asocia a los valores medios del test.

- La equidad, que se aproxima mediante el grado de dependencia de los resultados educativos con el contexto familiar.

- La calidad, que se relaciona con el porcentaje de alumnos de alto y bajo nivel.

Estas tres variables se recogen dentro de un indicador llamado: Índice de Desarrollo Educativo y dan una idea de la importancia que ha adquirido la equidad y la inclusión en educación, ya que se asocia directamente con la calidad educativa.

La educación inclusiva, actualmente, se considera como un proceso que surge de la necesidad individual del alumnado por sentirse reconocido y tomado en consideración, en un grupo de referencia. Su importancia radica en la necesidad de alcanzar un aprendizaje de calidad acorde a las capacidades del alumnado, profundizando en las intervenciones que resultan más efectivas para la obtención de un aprendizaje significativo (Eslava-Suanes, De León-Huertas, \& González-López, 2017). Es decir, tal y como establece la UNESCO (2015) estamos ante una educación inclusiva y equitativa de calidad y un aprendizaje que es para todos a lo largo de toda la vida. Según Muntaner (2019), esta idea está cada vez está más extendida, pues lo que se busca es el reconocimiento de las personas con sus vulnerabilidades, como seres activos y capaces de desarrollarse en una sociedad justa que les ofrezca a todos las mismas oportunidades.

Tal y como sostienen Ainscow, Booth y Dyson (2006), la inclusión educativa se apoya en tres referentes decisivos, como son la presencia, la participación y el progreso.

Hablar de presencia implica asegurar la participación de todo el alumnado en las experiencias de aprendizaje planteadas en el aula. La participación se define como la posibilidad de que todos los estudiantes formen parte de todas las actividades planificadas, teniendo presentes sus capacidades y potencialidades. Por progreso entendemos, la oportunidad concedida al alumnado para avanzar en su proceso formativo gracias a las experiencias de aprendizaje, que deben ser enriquecedoras y orientadas al desarrollo integral. 
A pesar de ser el objetivo de las últimas reformas educativas, su puesta en marcha no resulta fácil, ya que supone una aceptación de la diversidad, el replanteamiento de metodologías flexibles y abiertas, agrupamientos heterogéneos y los apoyos necesarios para facilitar la participación y el aprendizaje de todo el alumnado.

La inclusión se encuentra también dentro de la tendencia actual en investigación e innovación educativa; encaminada hacia la mejora de la práctica docente de forma que repercuta en todos y cada uno de los alumnos, garantizando así, una enseñanza de calidad para la totalidad de discentes (Sola, 2007).

\subsection{Conceptos de inclusión y exclusión}

Resulta conveniente aclarar los conceptos de inclusión y exclusión, ya que a lo largo de la historia educativa ha tenido varias acepciones.

El término inclusión engloba distintos aspectos del alumnado:

- El que presenta dificultades de aprendizaje y NEAE en general.

- Los que pertenecen a comunidades que se encuentran en situación de vulnerabilidad social.

En educación se relaciona con el concepto de equidad e igualdad de oportunidades para todo el alumnado indistintamente de sus características y circunstancias (García, 2015).

Ainscow y Miles (2008) refuerzan esta definición atendiendo a los siguientes aspectos:

- La relación con la discapacidad y las NEAE.

- La respuesta a las exclusiones disciplinarias.

- Los grupos vulnerables a la exclusión.

- La promoción de una escuela para todos educando para todos.

Por otro lado, García (2015) distingue dos tipos de equidad en educación:

En primer lugar, estaríamos ante el concepto débil, que se corresponde con el surgimiento de este término, el cual hace referencia a la equidad educativa entendida como sistemas de becas y ayudas disponibles para todos para, así, hacer posible la igualdad de oportunidades. Esto se lleva a cabo con el objetivo de que el alumnado alcance los estándares educativos previstos por la ley y obtenga una igualdad de trato. 
En segundo lugar, encontramos el concepto fuerte, en este caso se refiere a que todo el alumnado, independientemente de sus circunstancias particulares, alcance un buen rendimiento académico. Es este concepto fuerte el que está en continuo desarrollo (Ainscow \& Miles, 2008).

En esta misma línea, Mancebo y Goyneche (2010) también definen dos tipos de equidad educativa claramente diferenciados. Por un lado, la equidad de acceso, referida a la igualdad de oportunidades en el "punto de partida", desde el que todos los estudiantes poseen el derecho de ingresar en un determinado nivel del sistema educativo. Por otro lado, la que se refiere a la igualdad de oportunidades en el "punto de llegada". Este concepto implica equidad en las condiciones de aprendizaje de forma que todos los estudiantes alcancen un buen rendimiento académico.

El sistema educativo necesita crear garantías para la igualdad de oportunidades de forma que todo el alumnado alcance los mismos resultados académicos sin excepción, atendiendo al concepto de equidad más comprometido.

Para conseguir una verdadera inclusión educativa, no basta solo con contar con unas leyes educativas que la contemplen, también son necesarios los recursos y apoyos de la administración. Que los centros educativos cuenten con un liderazgo inclusivo que disponga de una formación adecuada, que realice el papel de motor del cambio y se encuentre coordinado con resto del profesorado, que a su vez también debe disponer de una formación precisa para conocer las estrategias pedagógicas inclusivas más adecuadas y manifestar el compromiso de responsabilidad ética y moral con su alumnado (Murillo, Krichesky, Castro, \& Hernández, 2010; León, Crisol, \& Moreno, 2018).

Aunque se ha avanzado en el uso de formas de organización del aula más inclusivas; como la creación de nuevos modelos de agrupamientos flexibles en programas de atención a la diversidad y adaptaciones de las metodologías y currículum según las necesidades educativas del alumnado, existen todavía muchas carencias, como soluciones efectivas a los problemas derivados de la diversidad cultural en las aulas (Rodríguez-Fuentes, \& Fernández-Fernández, 2017)

Por otro lado, atendiendo a la definición del término "exclusión educativa", Mancebo y Goyeneche (2010) distinguen hasta cinco formas:

1. La referida al alumnado que no está escolarizado.

2. La que alude al abandono de la escuela pasada la fase obligatoria, sin haber llegado a alcanzar los estándares de aprendizaje previstos por la ley educativa.

3. La que afecta a los estudiantes escolarizados que no se encuentran motivados ni integrados en las actividades que realiza la escuela, quedando al margen de la comunidad educativa. Lo que se relaciona, actualmente, con una escolaridad de baja calidad. 
4. La que deriva de metodologías de enseñanza elitistas que sólo contemplan los intereses, perspectivas y necesidades de la parte del alumnado más aventajado que cumple con las exigencias educativas.

5. La que se refiere a las escuelas en las que los aprendizajes generales que asimila su alumnado son de baja relevancia, dando ventaja al alumnado que cuenta con otros medios para formarse o que tiene la posibilidad de acudir a otros centros educativos.

Según este mismo autor, los puntos del dos al cinco son los que se relacionan con el punto de llegada de la equidad educativa, y es donde el sistema educativo tiene que poner especial atención, con el objetivo de atajar estas formas de exclusión educativa.

\subsection{El papel de los docentes en la escuela inclusiva}

La inclusión educativa requiere la identificación y eliminación de barreras relacionadas con creencias y actitudes hacia este proceso. Existen tres tipos de barreras que entorpecen la participación, la convivencia y el aprendizaje (Eslava-Suanes, De León-Huertas, \& González-López, 2017):

- Barreras políticas, que aparecen cuando existen contradicciones en las normativas educativas

- Barreras culturales, que se relacionan con las concepciones y actitudes que se poseen de cara a la diversidad

- Barreras didácticas, que tienen que ver con metodologías que no contemplan la inclusión del alumnado.

Resulta evidente que la actitud del docente influye considerablemente en la forma de integrar y aceptar el concepto de diversidad, lo que afectará a las expectativas hacia la manera en la que el alumnado debe aprender, incrementando o mermando su autoestima y motivación. Ambos, aspectos fundamentales del aprendizaje.

Mancebo y Goyeneche (2010) afirman con preocupación, que la escuela en multitud de ocasiones opta por aceptar con resignación la exclusión en lugar de luchar contra ella. Debido a la costumbre de convivir con esta realidad, se llega a normalizar la situación al no encontrar estrategias adecuadas para combatirla. Ante tal situación, se cierran todas las posibilidades de mejora.

Este panorama desolador se complica aún más cuando aparece la resistencia por parte de muchos docentes a la hora de implementar en sus aulas el cambio, continuando así con la impartición de sus lecciones de la forma tradicional y homogénea para un alumnado diverso, contribuyendo al aumento de las diferencias y exclusión, puesto que dicho alumnado debería ser atendido adecuadamente en función de las necesidades o dificultades individuales que presente, como se viene expresando reiteradamente, en este trabajo. 


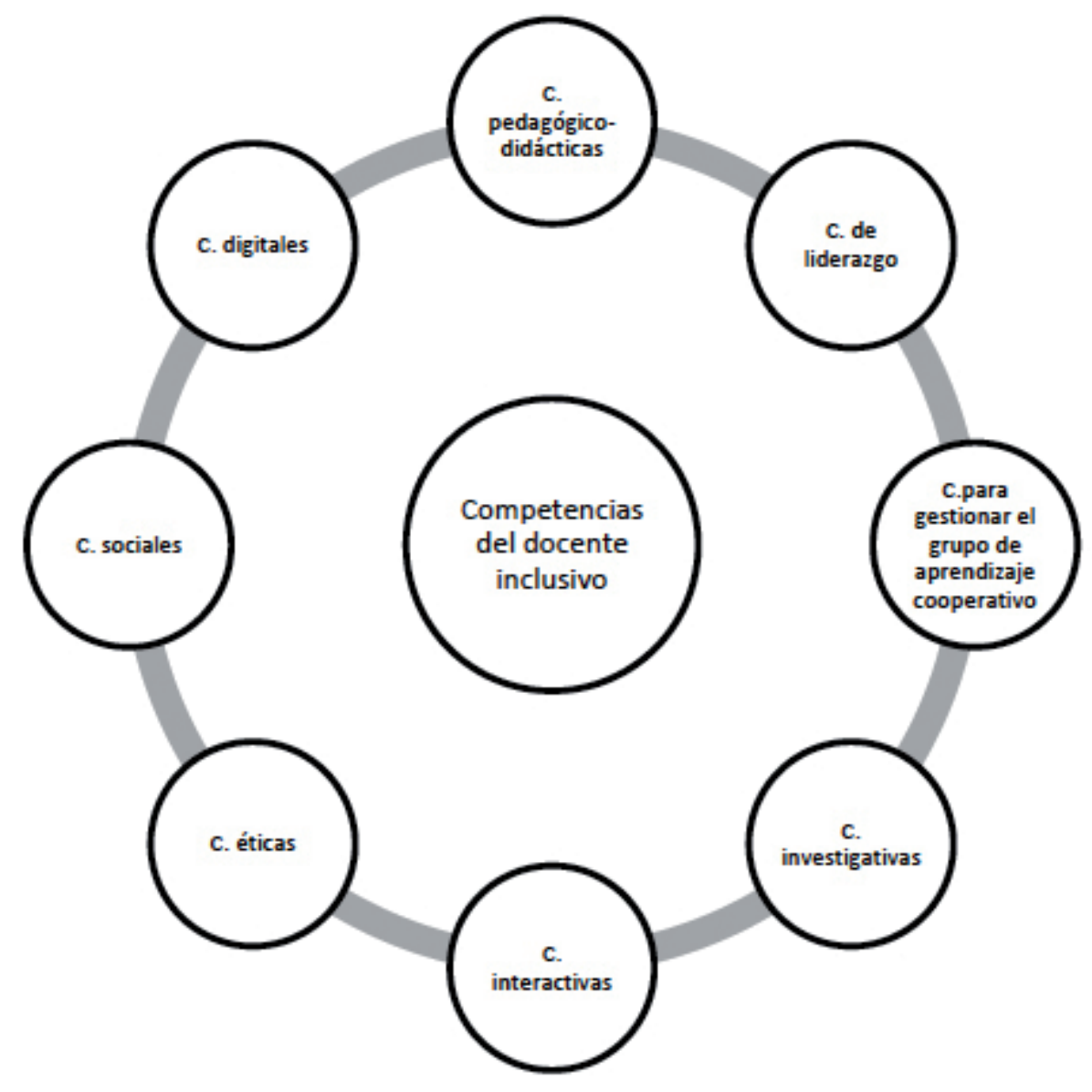

Figura 1. Adaptada de Fernández- Batanero (2012)

\section{Metodologías activas en pro de la inclusión educativa}

Educar en la actualidad no significa solo centrarse en los contenidos expresados en el currículo oficial, sino que hay muchos más elementos que se deben tener en cuenta. Es innegable, que en las últimas décadas se pone el foco de atención en uno de estos elementos como fundamental en los procesos de E-A. Nos referimos a las nuevas tecnologías de la información y la comunicación. Los nuevos recursos y dispositivos digitales surgidos recientemente son numerosos y son tan aceptados como rechazados por los miembros de la comunidad educativa (Marín-Díaz, 2015).

La renovación de las metodologías en todos los niveles educativos supone la introducción de las metodologías activas, las cuales implican una participación activa, como su propio nombre indica, por parte del estudiantado en todo su proceso formativo (Álvarez, González, Alonso, \& Arias, 2014; Roble- 
do, Fidalgo, Arias, \& Álvarez, 2015). Las metodologías activas se basan en la asunción del papel del profesor como guía para que el alumno asuma una autonomía en los procesos de E-A, para que así adquiera las competencias pertinentes y todos los tipos de saberes (conceptual, procedimental, actitudinal).

Este tipo de metodologías requieren la realización de un diseño claro, riguroso y viable del plan que se va a llevar a cabo con el alumnado y exige una alta implicación por parte del profesorado, ya que en sí mismas no garantizan el aprendizaje ni unos buenos resultados. El profesorado debe contar también, con la formación adecuada y ser capaz de adaptar la práctica al contexto del aula (Aróstegui-Barandica, \& Darretxe-Urrutxi, 2016).

De esta manera, favorecer la curiosidad hacia el aprendizaje de nuevos contenidos; adaptarse a los ritmos de aprendizaje del alumnado; motivar y generar un buen clima en el aula, contribuyendo así, a la atención a la diversidad y, por tanto, a la inclusión (Cascales-Martínez, \& Carrillo-García, 2018).

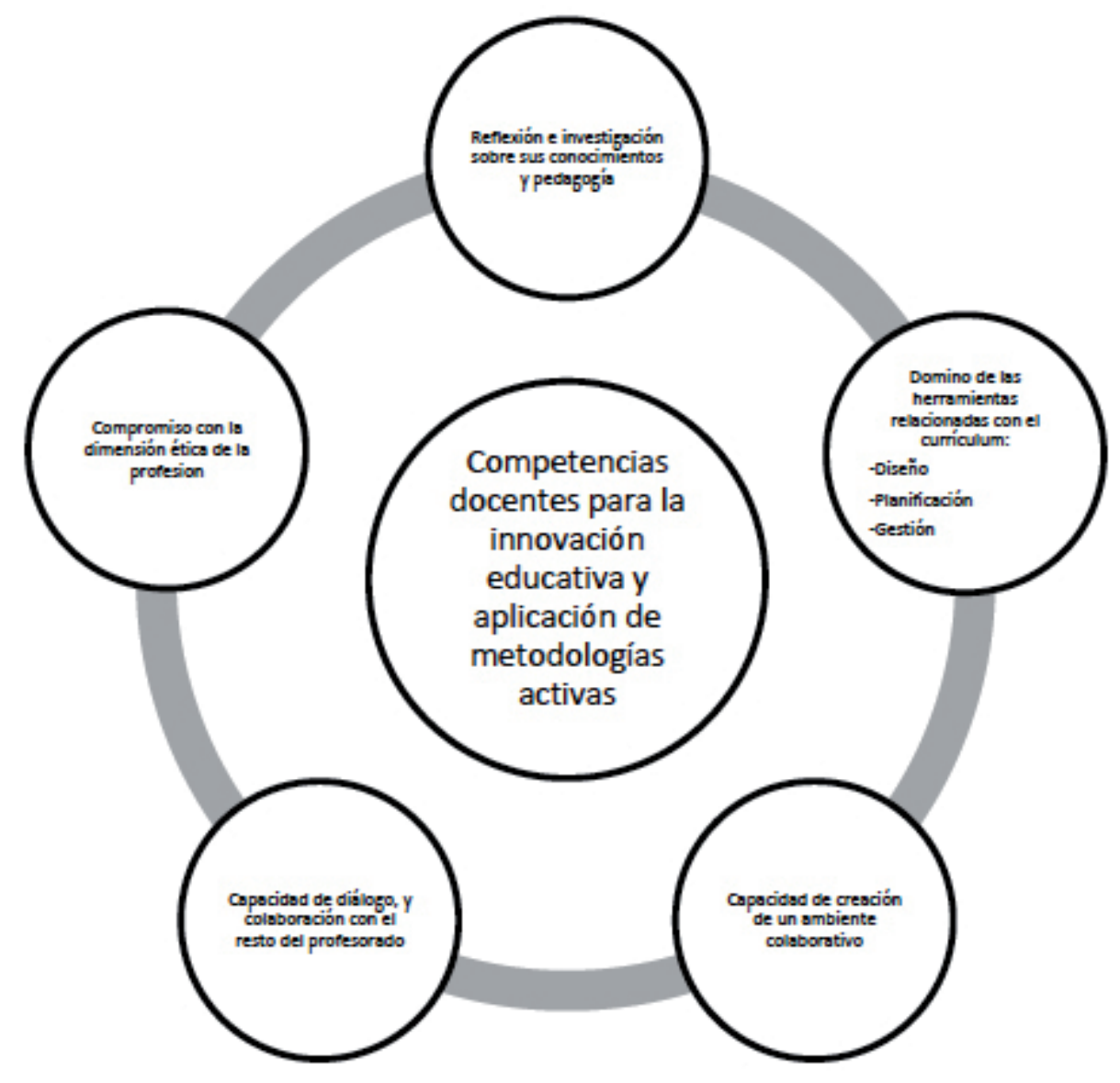

Figura 2. Adaptada de Guerrero y Moya (2011) 
El respeto de los ritmos de aprendizaje, según estos mismos autores, favorece el concepto de justicia social que hace que el aula se convierta en un espacio democrático e inclusivo, independientemente de las características del alumnado y de sus necesidades educativas. De esta forma el aula pasa a ser un espacio para crecer como ser social, que permite el desarrollo de las habilidades comunicativas y la expresión, donde se comparte y se aprende a vivir y convivir. En estos entornos de aprendizaje se fomenta también la colaboración entre el alumnado y se aprende desde la práctica activa.

Según Guerrero y Moya (2011), este cambio de metodología supone para el docente tener adquiridas algunas competencias profesionales que hagan posible este nuevo perfil y que se fundamentan en el dominio del conocimiento de la disciplina y en la investigación e innovación de la propia práctica docente. Las cuales guardan bastante relación con las competencias inclusivas citadas anteriormente (Ver Figura 2).

Estas metodologías van orientadas a dotar al alumnado de la capacidad de aprender por su cuenta dentro de un grupo de trabajo. Lo que se corresponde con un proceso de "aprender a aprender" que promueve la autonomía responsable del futuro profesional, y que dota de habilidades para saber localizar, evaluar, gestionar y sintetizar la información, con la finalidad de adaptarse con éxito los cambios de la sociedad del conocimiento.

\section{La gamificación en las aulas inclusivas}

Entre las metodologías activas que destacan por su relevante efectividad en el aprendizaje significativo e inclusivo se encuentra la gamificación. Esta estrategia educativa para diversos autores (Contreras, \& Eguia, 2016; Marín-Díaz, 2015; Ortiz-Colón, Jordán, \& Agredal, 2018) consiste en unir el aspecto lúdico con el aprendizaje. Esto es, fomentar que los procesos de E-A sean efectivos gracias a la introducción de las dinámicas de los videojuegos, de forma que integren el contenido y la práctica de tal manera que se aumenten la creatividad, la motivación y el interés por la asignatura o temática en concreto.

El uso de dichas dinámicas en el aula da respuesta a la necesidad existente de aprovechar todas las ventajas de este recurso independientemente del contexto en que se aplique (Sánchez-Rivas, RuizPalmero, \& Sánchez Rodríguez, 2017; Werbach \& Hunter, 2012).

En los últimos años son diversos los estudios que versan sobre el aprendizaje basado en el juego (Contreras-Espinosa, 2016; Hernández-Horta, Monroy-Reza, \& Jiménez-García, 2018; López-Rodríguez, Avello-Martínez, Baute-Álvarez, \& Vidal-Ledo, 2018), y todos ellos exponen que estamos ante un recurso adecuado para el aula y que es la motivación la que se ve mayormente afectada de manera positiva. 
Esta metodología activa surge de la necesidad de los docentes de motivar y mejorar el compromiso del alumnado con su autoaprendizaje. Utilizar la gamificación en las aulas es una herramienta muy efectiva por su capacidad de enseñar y reforzar conocimientos de forma motivadora y autónoma, dando así respuesta a las demandas actuales (Contreras \& Eguia, 2016). Las dinámicas de este tipo de juegos que se utilizan en gamificación están diseñadas para incentivar a las personas a participar. De hecho, la gamificación se comenzó a aplicar como una herramienta de marketing por su poder para fidelizar clientes y diferenciarse de la competencia.Estos mismos autores definen gamificación (palabra derivada de "game"; juego en inglés) como la utilización de los principios del videojuego a otros procesos no considerados lúdicos, con el objetivo de mejorar la motivación y la participación.

Cortizo, et al., (2011), amplían esta definición, concretando el tipo de procesos en los que se aplica la gamificación como una web, servicio, comunidad, contenido o campaña. Si lo trasladamos al contexto del aula, supone para el alumnado una serie de retos de aprendizaje que al alcanzarlos, les genera una recompensa inmediata adecuada al esfuerzo y a la complejidad de la tarea realizada. Es importante remarcar que la gamificación aunque es perfectamente compatible con la tecnología, no precisa de la presencia de ningún artefacto tecnológico para llevarla a cabo.

El sentimiento de plenitud que alcanza el alumnado jugando hace que aumente la concentración facilitando el aprendizaje. Esto será así, siempre y cuando se tengan en cuenta una serie de circunstancias (Aguilera, Fúquene, \& Ríos, 2014):

- El alumno tiene que estar en posesión de las capacidades necesarias para llevar a cabo la tarea propuesta

- El nivel del reto a realizar debe encontrarse dentro del nivel posible de actuación del alumnado

- La retroalimentación aportada por el juego debe ser inmediata, significativa y acorde al esfuerzo

Siguiendo unos diseños de gamificación propuestos por diversos autores (Arévalo, Mon, Batet, \& Catasús, 2015; Batet, 2016; Cortizo-Pérez et al, 2011; González, Jiménez, \& Moreira, 2018; Pérez, Rivera, \& Delgado, 2017; Pérez, Rivera, \& Trigueros, 2017) se pueden establecer una serie de características referentes a la gamificación que clarifican el concepto en sí y los elementos que implican:

- La ambientación: este elemento es de gran relevancia pues sobre él se configuran las historias, y los diferentes caminos que pueden realizar los alumnos cuando están jugando, es el hilo conductor que aporta el sentido al juego (Pérez, Rivera, \& Trigueros, 2017).

- Los juegos de rol: normalmente estas metodologías configuran dos realidades paralelas, la vida real del aula y una paralela que desarrolla en el juego, lo que supone que los alumnos adopten un rol que deben representar de manera individual o en grupo. 
- El compromiso: al inicio del videojuego o juego mediante el cual se va a aprender, esto hace que los alumnos se comprometan a aprovechar la experiencia de la mejor manera posible.

- Objetivos: el establecimiento de metas u objetivos es algo fundamental en este tipo de dinámicas. Que los participantes las conozcan desde el inicio supondrá que todas las acciones realizadas se encaminen hacia la consecución de estas.

- Con el objetivo de incentivar la motivación: se realizan acciones como las de recolección, que consiste en proporcionar premios de diversa índole cuando se alcanzan pequeños logros.

- Los puntos: son una manera simple de ofrecer retroalimentación a los usuarios sobre cómo van avanzando en el juego. Este elemento no solo motiva, sino que permite hacer comparaciones entre los diferentes participantes lo cual en ocasiones es positivo por la importancia que otorga estar en los primeros puestos. Dependiendo de la importancia de cada acción se le asignará una puntuación mayor o menor.

- La superación de los juegos o desafíos propuestos, así como la obtención de un determinado número de puntos: supondrá poder establecer diferentes niveles o bloques que, de nuevo, sitúan el logro de los usuarios en el juego.

- Estas dinámicas se realizan en pequeños grupos y están caracterizadas por la cooperación entre los participantes.

- Pero al mismo tiempo, el trabajo autónomo, en mayor o menor grado, es fundamental, para la consecución de los objetivos del grupo.

Además de estas características, otros autores (Aretio, 2016; Cortizo-Pérez et al., 2011; Oliva, 2016) han planteado una serie de ventajas a la hora de aplicar la gamificación en el ámbito educativo. Entre ellas destacan:

- Estimulación del esfuerzo para el alumnado

- Aumento del interés de los discentes por la materia

- Retroalimentación positiva mostrando los fallos y las áreas de mejora

- Fomento del trabajo del profesorado en el aula

- Posibilidad de ejercer un control más exhaustivo del nivel del alumnado en menos tiempo.

- Permite alcanzar los objetivos expresados en el currículum oficial a través del favorecimiento de la inclusión del alumnado. 


\section{La inclusión educativa a través del uso de la gamificación en el aula}

Los sistemas tecnológicos y las dinámicas lúdicas han irrumpido en el ámbito educativo favoreciendo a la diversidad del aula en su totalidad (Vidal-Esteve, López-Marí, Marín-Suelves, \& PeiratsChacón, 2018).

El objetivo que se persigue en el caso, por ejemplo, de personas con discapacidad intelectual, es que sean más autónomas y que se sientan incluidas en su entorno social. Según estos autores, la gamificación lo posibilita al desarrollar:

- Las habilidades comunicativas

- La interacción social

- La expresión de emociones

- La función simbólica

- La capacidad de resolución de problemas

- La participación en su propio aprendizaje

- La creatividad y el aspecto lúdico

González, et al. (2014), también comparten la misma opinión sobre los beneficios de la aplicación de la gamificación en el caso del alumnado con síndrome de Down. Dejaron constancia de ello, en una investigación que llevaron a cabo a través de un proyecto de intervención para mejorar la calidad de vida de este alumnado introduciendo la práctica deportiva en sus hábitos cotidianos a través de la gamificación.

Por lo tanto, la incorporación de las TIC en general, y de la gamificación en particular en un aula con alumnos con dificultades o NEAE, suponen una mejora del acceso a la información, la facilitación de la relación con su entorno y una mejora de la calidad de vida tanto en su plano académico como personal, entre otras muchas cosas (Rodríguez \& Arroyo, 2014).

De forma global se puede decir que los docentes que hacen uso de la gamificación en el aula con alumnado NEAE aprecian mejoras en una serie de aspectos relacionados con su aprendizaje (SánchezRivas, Ruiz-Palmero \& Sánchez-Rodríguez 2016), entre las que se destacan las siguientes:

- Aprovechamiento mayor y más eficaz del tiempo en las tareas de clase.

- Incremento de la motivación de forma considerable.

- Mayor rendimiento. 
- Aumento de la implicación con la materia y con el aprendizaje en general. El proceso educativo sale de las aulas y se extiende a todos los contextos del alumnado.

- Mejora de la autoestima, elemento necesario para el bienestar general y para la asimilación de los aprendizajes adecuadamente (Castro, 2012).

Estas dinámicas que presentan los videojuegos, como reiteran también Rodríguez y Arroyo (2014), suponen una estrategia fundamental en los procesos de E-A para el desarrollo de los aspectos relacionados con la inclusión, como son la competencia social y cívica. Así, posibilitan la individualización de los procesos cognitivos dando cabida a los diferentes ritmos y formas de aprendizaje y de comunicación. De esta forma, se disminuyen las brechas que se crean entre el alumnado debido a la diversidad, y se contribuye a disminuir la exclusión y la marginalidad que muchas veces sufre el alumnado con NEAE, logrando un cambio en la actitud y permitiendo que su aprendizaje resulte una experiencia gratificante.

En la siguiente tabla (Tabla 1) se realiza una comparativa entre las características que debe tener un aula inclusiva y las ventajas que aporta la gamificación a la equidad educativa, donde se pueden observar las coincidencias que hacen de esta metodología activa una herramienta idónea para la inclusión del alumnado

Tabla 1. Comparativa del aula inclusiva y el uso de la gamificación

\begin{tabular}{|c|c|}
\hline $\begin{array}{c}\text { APORTACIONES DEL USO } \\
\text { DE LA GAMIFICACIÓN EN EL AULA }\end{array}$ & $\begin{array}{l}\text { ACCIONES QUE FAVORECEN LA INCLUSIÓN } \\
\text { EN EL AULA }\end{array}$ \\
\hline Aumento de la motivación del alumnado & $\begin{array}{l}\text { Incrementar la motivación de todo el alumnado inde- } \\
\text { pendientemente de sus características particulares }\end{array}$ \\
\hline $\begin{array}{c}\text { Incrementa del rendimiento académico con la } \\
\text { mejora de la concentración que se consigue con los } \\
\text { juegos }\end{array}$ & $\begin{array}{l}\text { Aumentar el rendimiento de la totalidad del alumnado } \\
\text { para que alcancen los mismos objetivos educativos }\end{array}$ \\
\hline Permite la participación activa del alumnado & $\begin{array}{l}\text { Permitir la participación activa del alumnado, abando- } \\
\text { nando su papel pasivo tradicional }\end{array}$ \\
\hline Desarrollo de la autonomía & $\begin{array}{l}\text { Fomentar la autonomía de todo el alumnado en general } \\
\text { y del alumnado con NEAE en particular }\end{array}$ \\
\hline $\begin{array}{l}\text { El alumnado se convierte en el constructor de su } \\
\text { propio aprendizaje }\end{array}$ & $\begin{array}{c}\text { Favorecer que el alumnado construya su propio } \\
\text { aprendizaje }\end{array}$ \\
\hline Mejora la autoestima & $\begin{array}{l}\text { Mejorar la autoestima de todo el alumnado, especial- } \\
\text { mente del que tiene dificultades de aprendizaje, ya que } \\
\text { tiende a tenerla baja }\end{array}$ \\
\hline
\end{tabular}




\begin{tabular}{|c|c|}
\hline $\begin{array}{c}\text { Respeta los distintos ritmos de aprendizaje que se } \\
\text { encuentran en el aula }\end{array}$ & $\begin{array}{c}\text { Respetar los distintos ritmos de aprendizaje individuales } \\
\text { para contribuir a la justicia social }\end{array}$ \\
\hline $\begin{array}{l}\text { Los aprendizajes se contextualizan siendo más } \\
\text { significativos por su puesta en práctica }\end{array}$ & $\begin{array}{l}\text { Contextualizar los aprendizajes y realizar las distintas } \\
\text { tareas desde la práctica }\end{array}$ \\
\hline $\begin{array}{l}\text { Favorece la adquisición de las competencias social } \\
\text { y cívica }\end{array}$ & Mejorar la competencia social y cívica \\
\hline Mejora las habilidades comunicativas & Fomentar la comunicación \\
\hline $\begin{array}{l}\text { La experiencias lúdicas resultan muy agradables y } \\
\text { divertidas }\end{array}$ & $\begin{array}{l}\text { Realizar experiencias de aprendizaje agradables y } \\
\text { divertidas }\end{array}$ \\
\hline $\begin{array}{l}\text { El alumnado realiza actividades de colaboración en } \\
\text { grupo }\end{array}$ & Fomentar la colaboración entre el alumnado \\
\hline $\begin{array}{c}\text { El clima del aula se ve favorecido por el ambiente } \\
\text { comunicativo y distendido que genera esta metodo- } \\
\text { logía lúdica }\end{array}$ & Obtener un clima del aula positivo \\
\hline $\begin{array}{c}\text { Desarrolla la capacidad de resolver problemas en } \\
\text { distintos contextos }\end{array}$ & Fomentar la capacidad de resolución de problemas \\
\hline Permite la posibilidad de expresar emociones & Permitir la expresión de emociones \\
\hline Se premia el esfuerzo & Estimular el esfuerzo \\
\hline
\end{tabular}

Notas: Elaboración propia

A partir de estas aportaciones, es posible comprobar que las similitudes entre los beneficios que supone el uso de la gamificación en el aula y los elementos necesarios para que un aula sea inclusiva, son numerosas. Esto es, la gamificación no solo aporta elementos lúdicos y motivadores sino que supone la implementación de algo fundamental en los centros escolares, la inclusión.

\section{Conclusiones}

Con la introducción de la gamificación en los centros educativos se cambian los modos en los que se habían percibido hasta ahora las metodologías y los procesos de E-A. Teniendo en cuenta todas las propuestas y tendencias de los últimos tiempos, se puede afirmar que la gamificación se ajusta perfecta- 
mente a la respuesta inclusiva que la escuela demanda en cualquier etapa educativa y que las leyes actuales exigen para atender a las necesidades que la sociedad actual espera del alumnado. Sin embargo, se debe tener presente que todavía existen resistencias por parte de los centros y del profesorado a la hora de implementar esta nueva tendencia, debido al cambio tan radical de rol que debe asumir el profesorado y las nuevas competencias que debe potenciar para llevar a cabo la nueva práctica docente.

Con este trabajo se pretende demostrar que el uso de la gamificación dentro de las aulas, como elemento favorecedor y potenciador del aprendizaje del alumnado, independientemente de la materia del currículo que se trate, se ajusta perfectamente al logro de la equidad educativa que nuestro sistema educativo ansía. Estamos asistiendo al auge de esta tendencia, ya que no son pocos los documentos de la literatura científica que defienden las potencialidades y ventajas que se pueden extraer de la gamificación.

A lo largo de este artículo se ha llegado a la conclusión, tras conocer las necesidades inclusivas de la educación y las características de las metodologías activas, que el uso de la gamificación es una herramienta a disposición de los docentes que supone un antes y un después en la inclusión de todos y cada uno de los alumnos, exponiendo sus ventajas y potencialidades para el aula inclusiva.

La conjugación de la inclusión y la gamificación supone paliar la exclusión educativa generada por diversos motivos, además de un aumento de la motivación del alumnado que se implica más en su proceso de E-A; del mismo modo, favorece competencias como la social y cívica, entre otras muchas (Monroy-Reza \& Jiménez-García, 2018; Rodríguez \& Arroyo, 2014).

Por lo tanto, ha quedado patente a lo largo de todo el trabajo que aunque hacen falta numerosos avances para la correcta aplicación de esta dinámica en las aulas, como por ejemplo formativos por parte del profesorado, o de dotación de recursos y herramientas, las ventajas expuestas junto con los buenos resultados obtenidos al llevar a cabo estas prácticas, no hacen sino animar a los docentes a emplear la gamificación en cualquier etapa para un favorecimiento del aprendizaje significativo sin exclusión.

\section{Referencias}

Aguilera, A., Fúquene, C. \& Ríos, W. (2014). Aprende jugando: El Uso de Técnicas de Gamificación en Entornos de Aprendizaje. IM-Pertinente, 2(1), 125-143

Ainscow, M., Booth, T., \& Dyson, A. (2006). Improving schools, Developing Inclusion. Nueva York. Routledge.

Ainscow, M. \& Miles, S. (2008) Por una educación para todos que sea inclusiva ¿Hacia dónde vamos? Perspectivas 1, Madrid 
Aretio, L. G. (2016). El juego y otros principios pedagógicos. Su pervivencia en la educación a distancia y virtual. RIED. Revista Iberoamericana de Educación a Distancia, 19(2), 9-23.

Aróstegui-Barandica, I., \& Darretxe-Urrutxi, L. (2016). Estrategias metodológicas activas en la asignatura de "Bases de la Escuela Inclusiva" de la EU de Magisterio de Bilbao. REDU. Revista de Docencia Universitaria, 14(2), 315-340.

Álvarez, A., González, J., Alonso, J, \& Arias, J. (2014). Indicadores centinela para el plan de Bolonia. Revista de Investigación Educativa, 32(2), 327-338. doi: 10.6018/rie.32.2.172721

Brasó i Rius, J., \& Torrebadella Flix, X. (2018). Reflexiones para (re) formular una educación física crítica. Revista Internacional de Medicina y Ciencias de la Actividad Física y del Deporte. doi: 10.15366/rimcafd2018.71.003

Cálciz, A. B. (2011). Metodologías activas y aprendizaje por descubrimiento. Revista digital innovación y experiencias educativas.

Cascales-Martínez, A., \& Carrillo-García, M. E. (2018). Aprendizaje basado en proyectos en educación infantil: cambio pedagógico y social. Revista Iberoamericana de Educación, 76, 79-98

Castro, C. de (2012). El futuro de las tecnologías digitales aplicadas al aprendizaje de personas con necesidades educativas especiales. Revista de Educación a Distancia, 32, 1-43.

Coleman, J. S. (1968). Equality of educational opportunity. Integrated Education, 6(5), 19-28.

Contreras, R. S. \& Eguia, J.L. (2016). Gamificación en Aulas Universitarias. Bellaterra: Institut de la Comunicació, Universitat Autònoma de Barcelona

Contreras-Espinosa, R. (2016). Juegos digitales y gamificación aplicados en el ámbito de la educación. RIED. Revista Iberoamericana de Educación a Distancia, 19 (2), 27-33.

Corchuelo, C. A. (2018). Gamificación en educación superior: experiencia innovadora para motivar estudiantes y dinamizar contenidos en el aula. Edutec. Revista Electrónica de Tecnología Educativa, (63), 29-41. doi: https://doi.org/10.21556/edutec.2018.63.927

Cortizo-Pérez, J. C., Carrero-García, F. M., Monsalve-Piqueras, B., Velasco-Collado, A., Díaz del Dedo, L. I., \& Pérez-Martín, J. (2011). Gamificación y Docencia: Lo que la Universidad tiene que aprender de los Videojuegos. Abacus. Universidad Europea de Madrid. 
Eslava-Suanes, M. D, de León Huertas, C., \& Gonzáles- López, I. (2017). La formación en competencias transversales para trabajar en entornos educativos inclusivos. Revista de Educación Inclusiva, 8(2), 58-76

Fernández, J.M. (2012). Capacidades y competencias docentes para la inclusión del alumnado en la educación superior. Revista de la educación superior, 41(162), 9-24.

Fernández-Batanero, J.M. (2018). TIC y la discapacidad. Conocimiento del profesorado de Educación Especial. ICT and Disability. Knowledge of the Special Education teachers. Aportaciones arbitradas. Revista Educativa Hekademos, 24, 19-29.

García, J. E. (1996). Equidad y Educación en Chile. Santiago: Mimeo.

García, R. (2015). Desafíos a la igualdad en el siglo XXI: Dificultades y retos de la escuela comprensiva. Revista Española de Educación Comparada, 26 135-152. doi: 10.5944/ reec.26.2015.14445.

González, C. S., Mora, A., Moreno, L., \& Socas, M. C. (2014). Actividad física y síndrome de down: un enfoque gamificado basado en TIC. Comunicación presentada en: III Jornadas de buenas prácticas en atención a la diversidad: ¿Qué aportan las TIC? Tenerife: Universidad la Laguna.

González, L. E. Q., Jiménez, F. J., \& Moreira, M. A. (2018). Más allá del libro de texto. La gamificación mediada con TIC como alternativa de innovación en Educación Física. Retos: nuevas tendencias en educación física, deporte y recreación, (34), 343-348.

Guerrero, M. J. L., \& Moya, E. C. (2011). Diseño de cuestionarios (OPPUMAUGR y OPEUMAUGR): La opinión y la percepción del profesorado y de los estudiantes sobre el uso de las metodologías activas en la universidad. Profesorado. Revista de Currículum y Formación de Profesorado, 15(2), 271-298.

Hernández-Horta, I. A., Monroy-Reza, A., \& Jiménez-García, M. (2018). Aprendizaje mediante Juegos basados en Principios de Gamificación en Instituciones de Educación Superior. Formación universitaria, 11(5), 31-40.

Jerez, O. (2015). Aprendizaje activo, diversidad e inclusión. Enfoque, metodologías y recomendaciones para su implementación. Ediciones Universidad de Chile 
León, M. J., \& Crisol, E. (2011). Diseño de cuestionarios (OPPUMAUGR y OPEUMAUGR): La opinión y la percepción del profesorado y de los estudiantes sobre el uso de las metodologías activas en la universidad. Profesorado. Revista de Currículum y Formación de Profesorado, 15(2), 271-298.

León, M.J., Crisol, E., \& Moreno, R. (2018) Las Tareas del Líder Inclusivo en Centros Educativos de Zonas Desfavorecidas y Favorecidas. Revista Iberoamericana sobre Calidad, Eficacia y Cambio en Educación, 16(2), 21-40. doi: https://doi.org/10.15366/reice2018.16.2.002

Lobato, C., \& Madinabeitia, A. (2011). Perfiles motivacionales del profesorado ante la formación en metodologías activas en la universidad. Formación universitaria, 4(1), 37-48. doi: 10.4067/S0718-50062011000100006

López-Rodríguez, I., Avello-Martínez, R., Baute-Álvarez, L. M., \& Vidal-Ledo, M. (2018). Juegos digitales en la educación superior. Educación Médica Superior, 32(1), 0-0.

Marín-Díaz, V. (2015). La gamificación educativa. una alternativa para la enseñanza creativa. Digital Education Review, (27).

Mancebo, M. E., \& Goyeneche, G. (2010). Las políticas de inclusión educativa: Entre la exclusión social y la innovación pedagógica. VI Jornadas de Sociología de la UNLP, 9 y 10 de diciembre de 2010, La Plata, Argentina. En Memoria Académica.

Ministerio de Educación, Cultura y Deporte (3, mayo 2006). Ley Orgánica 2/2006, de 3 de mayo de Educación. Recuperado de_https://www.boe.es/buscar/pdf/2006/BOE-A2006-7899-consolidado.pdf

Muntaner, J.J. (2019). La inclusión una apuesta social y educativa, en El Homrani, M., Arias, S.M. \& Ávalos, I. (Coords). La inclusión: una apuesta educativa y social. (pp. 41-57). Madrid: Wolters Kluwer.

Murillo, F.J.; Krichesky, G., Castro, A.M. y Hernández, R. (2010). Liderazgo para la inclusión escolar y la justicia social. Aportaciones de la investigación. Revista Lationamericana de Educación Inclusiva, 4(1), 169-186.

Oliva, H. A. (2016). La gamificación como estrategia metodológica en el contexto educativo universitario. Realidad y Reflexión, 16(44), 108-118. 
Ortiz-Colón, A. M., Jordán, J., \& Agredal, M. (2018). Gamificación en educación: una panorámica sobre el estado de la cuestión. Educ. Pesqui., São Paulo, 44. doi: http://dx.doi. org/10.1590/S1678-4634201844173773

Perazzo, G., \& Gargiulo, L. (2009). Informe warnock: Revisión y reflexión bioética a los 25 años de su publicación. Vida y Ética, 10(1), 19-25.

Pérez, I. J., Rivera, E., \& Delgado, M. (2017). Mejora de hábitos de vida saludables en alumnos universitarios mediante una propuesta de gamificación. Nutrición Hospitalaria, 34(4), 942-951.

Pérez, I. J., Rivera, E., \& Trigueros, C. (2017). La profecía de los elegidos: Un ejemplo de gamificación aplicado a la docencia universitaria. Revista Internacional de Medicina y Ciencias de la Actividad Física y el Deporte, 17(66), 243-260 doi: https://doi.org/10.15366/ rimcafd2017.66.003

Robledo, P., Fidalgo, R., Arias, O., \& Álvarez, L. (2015). Percepción de los estudiantes sobre el desarrollo de competencias a través de diferentes metodologías activas. Revista de Investigación Educativa, 33(2), 369-383. doi: https://doi.org/10.6018/rie.33.2.201381

Rodríguez-Fuentes, A., \& Fernández-Fernández, A.D. (2017). Adultos que conforman menores en una escuela de colores. Actitudes de progenitores y profesores ante la diversidad cultural. Revista de Investigación Educativa, 35(2), 465-482 doi: http://dx.doi.org/10.6018/ rie.35.2.256371

Rodríguez, M., \& Arroyo, M.L. (2014). Las TIC al servicio de la inclusión educativa. Digital Education Review, 25, 108-126.

Sánchez-Rivas, E., Ruiz-Palmero, J., \& Sánchez-Rodríguez, J. (2017). Videojuegos frente a fichas impresas en la intervención didáctica con alumnado con necesidades educativas especiales. Educar, 53(1), 29-48.

Serrano, J. M. \& Pons, R. M. (2011). El constructivismo hoy: enfoques constructivistas en educación. Revista Electrónica de Investigación Educativa, 13(1), 1-27. Recuperado de: http://redie.uabc.mx/index.php/redie/article/download/268/431

Sola, T. (2007). La escuela inclusiva: una respuesta educativa para todos. Gestionando los nuevos actores y escenarios de la formación en la sociedad del conocimiento 12(2) 89-108. 
UNESCO. (2015). Declaración de Incheón: de la aprobación a la aplicación. Recuperado de: https://es.unesco.org/news/declaracion-incheon-aprobacion-aplicacion

Vidal-Esteve, I., López-Marí, M., Marín-Suelves, D., \& Peirats-Chacón, J. (2018). Revisión y análisis de investigación publicada sobre intervención gamificada en discapacidad intelectual. Revista científica electrónica de Educación y Comunicación en la Sociedad del Conocimiento, 18(2), 274-297.

Villar, A. (2018). Rendimiento, equidad y calidad: el desarrollo educativo en España según PISA 2015. Cuadernos Económicos de ICE, (95), 79-97. doi: https://doi.org/10.32796/ cice. 20189

Werbach, K., \& Hunter, D. (2012). For the Win: How Game Thinking Can Revolutionize Your Business. Harrisburg: Wharton Digital Press. 